\title{
mm-VLBI: Jets in the Vicinity of Galaxy-Cores
}

\author{
T.P.Krichbaum ${ }^{1}$, K.J.Standke ${ }^{1}$, D.A.Graham ${ }^{1}$, A.Witzel ${ }^{1}$, C.J.Schalinski' ${ }^{2}$, J.A.Zensus ${ }^{3}$ \\ ${ }^{1}$ Mar-Planck-Institut für Radioastronomie (MPIfR), Bonn, Germany \\ ${ }^{2}$ Institut de Radio Astronomie Millimetrique (IRAM), Grenoble, France \\ 'National Radio Astronomy Observatory (NRAO), Socorro, N.M., USA
}

\begin{abstract}
Millimeter-VLBI provides an angular resolution of up to a few tens of microarcseconds and allows imaging of compact radio sources, self-absorbed at longer wavelengths, with unsurpassed angular resolution. At $43 \mathrm{GHz}$ the participation of the VLBA and the $30 \mathrm{~m}-\mathrm{MRT}$ at Pico Veleta (e.g. Krichbaum et al., $1993 \mathrm{a \& b}$ ), and at $86 \mathrm{GHs}$ the addition of the $100 \mathrm{~m}-\mathrm{RT}$ at Effelsberg and the $30 \mathrm{~m}$-MRT (Schalinski et al., 1993, and this volume) have improved the imaging capabilities of mm-VLBI observations.

Results: The increased sensitivity of mm-VLBI observations allows the investigation of fainter objects, previously not accessible. As one example we show in Fig.1 the first detection of the compact radio source $\mathrm{Sgr} \mathrm{A}^{*}$ in the Galactic Center with VLBI at $43 \mathrm{GHz}$ in May 1992 (Krichbaum et al., 1993d) and at $86 \mathrm{GHz}$ in April 1993 (Krichbaum et al., 1994). In both observations the size of $\mathrm{Sgr} \mathrm{A}^{*}$ appeared to be larger than its expected scattering size, indicative of intrinsic source structure showing up at mm-wavelengths. Future monitoring with mm-VLBI is necessary to search for (not unexpected) structural variability.

Monitoring of AGN with mm-VLBI reveals in all cases observed in sufficient detail jet curvatures of increasing amplitude towards the self-absorbed VLBI-cores (e.g. in 1803+784: Krichbaum, 1990, OJ 287: Krichbaum et al., 1993c), and sub- or superluminal motion along 'quasi-helically' bent trajectories (e.g. 3C 84: Krichbaum et al., 1993b; 3C 273: Krichbaum et al., 1993c), which differ sometimes for adjacent jet components (e.g. 3C 345: Krichbaum \& Witzel, 1992, Krichbaum et al., 1992\&1993a). In 3C 84, 3C 273 and 3C 345 the apparent velocity of jet components varies systematically along the jet axis, in $4 \mathrm{C} 39.25$ (Alberdi et al., 1993) a moving component decelerates and brightens, all of this suggesting differential Doppler boosting and motion along three-dimensionally curved trajectories. In 3C 345 the complex kinematics of C4 and C5 (Zensus, this volume) has been geometrically modeled by motion along a helical path on the surface of a conical jet (Qian et al., 1992, Steffen et al., 1993, and this volume; see also Camenzind, this volume). As a new example, the oscillations of the inner jet and its velocity variations $\beta_{\text {epp }}(r)$ are shown for the BL Lac object $1803+784$ in Fig. 2 (see the maps in: Krichbaum et al., 1993b). The frequent occurence of 'quasi-sinusoidal' bends in the inner jets of very different classes of AGN (QSO's, BL Lac's, Seyfert's) suggests that this effect is common in a large fraction of AGN and that the underlying jet-physical process may be fundamental for the understanding of the creation of jets.
\end{abstract}

Acknowledgements: We thank the staff of the observatories for their help. Special thanks for their help are due to S. Britzen, A.-M. Gontier, A. Greve, C. Naundorf, H. Steppe, R. Wegner, and W. Steffen. The work of T.P.K. was supported by the German BMFT-Verbundforschung.

\section{References}

Alberdi, A., Krichbaum, T.P., Marcaide, J.M., et al., $A \cup A, 271,93$.

Krichbaum, T.P., 1990, in: Parsec-scale radio jets, ed. J. A. Zensus and T. J. Pearson (Cambridge University Press), p. 83.

Krichbaum, T.P., and Witzel, A., 1992, in: Variability of Blazars, ed. E. Valtaoja and M. Valtonen (Cambridge University Press), p. 205.

Krichbaum, T.P., Witzel, A., Graham, D.A., Zensus, J.A., 1992, in: Physics of Active Galactic Nuclei, ed. W.J. Duschl and S.J. Wagner (Springer, Heidelberg), p. 574.

Krichbaum, T.P., Witzel, A., Graham, D.A., et al., 1993a, $A \& A, 275,375$.

Krichbaum, T.P., Witzel, A., Graham, D.A., et al., 1993b, in: Sub Arcsecond Radio Astronomy, ed. R.J. Davis and R.S. Booth, Cambridge University Press, p. 181.

Krichbaum, T.P., Witzel, A., Graham, D.A., 1993c, in: Jets in Extragalactic Radio Sources, ed. H.-J. Röser and K. Meisenheimer (Springer, Heidelberg), p. 71.

\section{7}

T. J.-L. Courvoisier and A. Blecha: Multi-Wavelength Continuum Emission of AGN, 187-188.

(C) 1994 IAU. Printed in the Netherlands. 


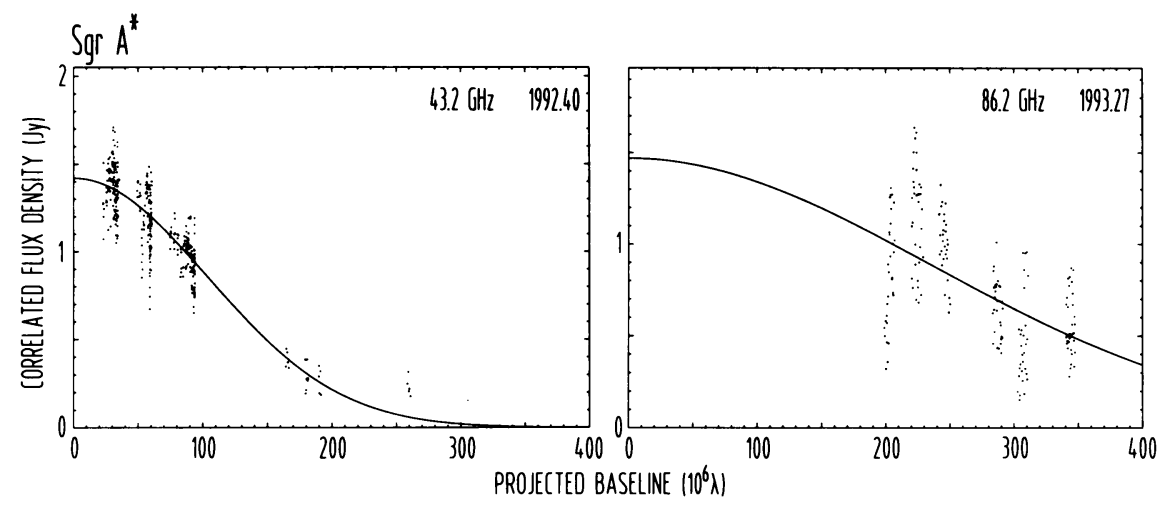

Fig. 1. Correlated flux density of $\mathrm{Sgr}_{\mathrm{A}} \mathrm{A}^{*}$ plotted versus projected uv-distance (left: at $43 \mathrm{GHs}$, right: at $86 \mathrm{GHz}$ ). The solid line represents a circular Gaussian component fit to the data with flux density and size (FWHM) of $S_{43} \mathrm{GHz}_{\mathrm{z}}=(1.42 \pm 0.10) \mathrm{Jy}, \theta_{43 \mathrm{GHz}}=(0.75 \pm 0.08) \mathrm{mas}$, respectively $S_{8 \mathrm{OH} z}=(1.47 \pm 0.75) \mathrm{Jy}, \theta_{80 \mathrm{OHz}}=(0.33 \pm 0.14)$ mas. The corresponding brightness temperatures are $T_{B}(43 G H z)=1.7 \cdot 10^{\circ} \mathrm{K}$, and $T_{B}(86 G H z)=2.2 \cdot 10^{\circ} \mathrm{K}$. The scattering sires extrapolated from VLBI observations at $\nu \leq 22 \mathrm{GHz}$ are $\theta_{45}^{\text {ecat }} \mathrm{Gz}=0.53 \pm 0.02 \mathrm{mas}$ and $\theta_{86}^{\text {ecat }} \mathrm{Gz}=0.13 \pm 0.01 \mathrm{mas}$, both smaller than the source sires given above (Krichbaum et al., $1993 \mathrm{~d} \& 1994)$. Note that at $r_{0}=8.5 \mathrm{kpc}$ an angle of 0.1 mas corresponds to $1.3 \cdot 10^{13} \mathrm{~cm}=0.9 \mathrm{AU}$.
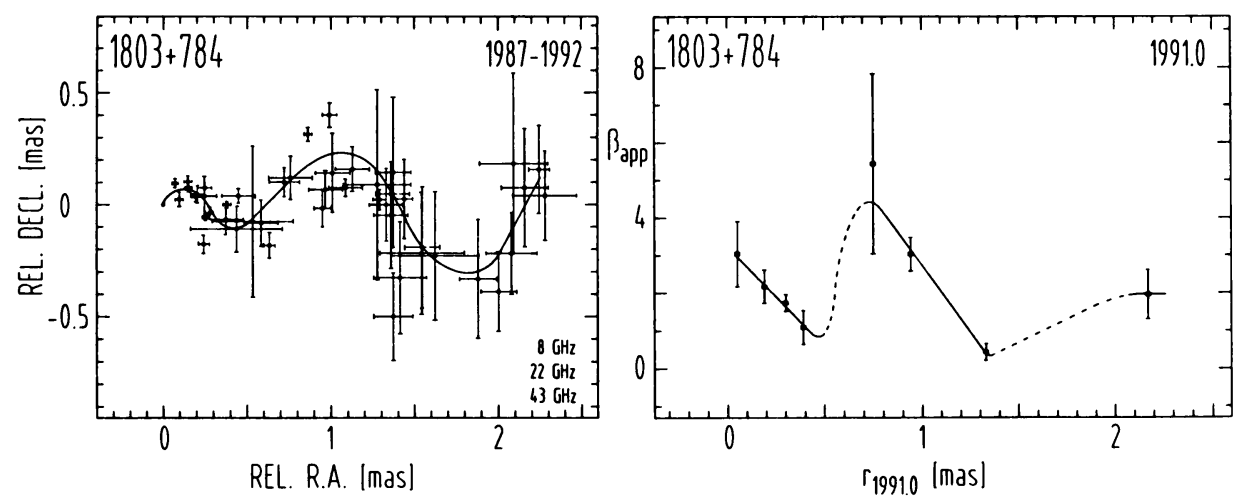

Fig. 2. Left: Relative positions of the VLBI components of the inner jet of $1803+784$, obtained between 1987-1992 at 8, 22, and $43 \mathrm{GHz}$ with respect to the stationary assumed VLBI-core. Data at $8 \mathrm{GHz}$ are from Britzen et al., this volume. Right: Apparent velocity $\beta_{\text {app }}(z=0.864$, $\left.H_{0}=100 \mathrm{~km} \mathrm{~s}^{-1} \mathrm{Mpc}^{-1}, q_{0}=0.5\right)$ of the jet components plotted versus core-separation at epoch 1991.0. The oscillations of the 'mean jet-axis' and the systematic variations of $\beta_{\text {app }}(r)$ along the jet strongly indicate motion along a three-dimensionally bent path, e.g. a helically bent jet.

Krichbaum, T.P., Zensus, J.A., Witzel, A., et al., 1993d, A\&A, 274, L37.

Krichbaum, T.P., Schalinski, C.J., Witzel, A., et al., 1994, in: The Nuclei of Normal Galaxies: Lessons from the Galactic Center, ed. R. Genzel (Kluwer, Dordrecht), in press.

Qian, S.J., Witzel, A., Krichbaum, T.P., et al., 1992, Chin. Astron. Astrophys, 16/2, 137.

Schalinski, C.J., Greve, A., Grewing, M., et al., 1993, in: Sub Arcsecond Radio Astronomy, ed. R.J. Davis and R.S. Booth, Cambridge University Press, p. 184.

Steffen, W., Krichbaum, T.P., Witzel, A., Zensus, J.A., in: Sub Arcsecond Radio Astronomy, ed.

R.J. Davis and R.S. Booth, Cambridge University Press, p. 363. 